\title{
Bamboo Timber Mildew and Anti-mold Technology
}

\author{
Liu yadi \\ College of Furniture and Art Design, Central South University of Forestry and Technology, \\ Changsha, 410004, China \\ cuckoo_lyd@163.com
}

\begin{abstract}
Keywords: bamboo timber, mold, mildew mechanism, anti-mold method
\end{abstract}
\begin{abstract}
China is one of the world's major bamboo producers, and the development and utilization of bamboo timber has become a new industry. But mildew often appears on both original bamboo substrate and bamboo processing material in the processes of storage, processing, transport and utilization, resulting in the pigment pollution of surface that affects bamboo quality and brings great losses to the enterprise. This paper describes the mildew mechanism of bamboo timber, the main causal fungi and the anti-mold technology, and also prospects the development direction of the research in future, which provided reference for the further study on the anti-mold technology of bamboo timber.
\end{abstract}

\section{Foreword}

China is rich in bamboo resources, bamboo is known as the second forest resources, and it is an important substitute of wood. Bamboo is qualified with great intensity, good toughness, good rigidity and relatively lighter color, moreover, it is easy to be bleached and dyed. Original bamboo is cut into thin bamboo chip, wood plywood, medium density fiberboard and particle board are used as substrate in veneer processing, which can replace the utilization of the timber of large diameter broad-leaved forest, to produce plates with various decorative effects. After bamboo was processed into a thin bamboo chip, its added value can be increased more than three times, if the bamboo is processed and assembled into the substrate of various decorative pendants, the economic benefits are more considerable. The anti-correction and anti-mold products of bamboo, as bamboo contains high level of carbohydrates and protein, it absorbs moisture easily and also is easily to be eroded by insect and bacteriaThe processing by using the preservative with a higher proportion of borate salts and PCP-Na class does not meet the requirements of environmental protection, and they have already been prohibited in some countries. With the rise of bamboo timber industry, the bamboo timber mold preventive and anti-mold technology with high efficiency and low toxicity need to be researched and developed urgently, which is significant in promoting the development of bamboo industry in China.

\section{Bamboo mildew and its breeding environment}

The mildew of bamboo timber is caused by the mold bred on its surface. Bamboo mildew fungi feed on the material without cell wall, so it has minor hazards to the cell and also does not affect the strength of bamboo. But the pigment secreted by colored mycelium and Paoyuhe mycelium will leave blue, brown or gray mildew on the bamboo surface, even resulting in a considerable depth of staining, and they cannot be removed by bleach treatment or surface planning. Through this, the bamboo loses its natural texture and color, which will greatly reduce the value of bamboo timber and bamboo products. Bamboo decay fungi can also secrete cellulose and hemicellulose hydrolysis enzyme. Therefore, bamboo decay fungi can obtain nutrients through the degradation of cellulose, hemicellulose or even lignin, and some other materials with cell wall, so that the bamboo cells are destroyed and the mechanical strength is reduced or even completely loses use value.

Mold damages bamboo timber through the growth of spore. Spore is equivalent to the seed of higher plant, it contains a single cell or combined cells and it is spread by wind, water, insect or the transport of damaged bamboo. Under proper temperature and humidity, spore will germinate the mycelium which produces single cell, the mycelium invades the bamboo timber to absorb sugars, starch and some other raw materials of bamboo cell, and then it grows on the bamboo ${ }^{[1]}$. 
The structure and chemical composition of bamboo provide sufficient material conditions for the breeding and growth of the fungus, but the breeding of fungus also requires certain conditions like temperature, moisture, $\mathrm{PH}$, etc. Weng Yuexia, etc. ${ }^{2]}$ propose that the decisive factor of bamboo mildew is environmental humidity, when the relative humidity of environment is below $75 \%$, no mildew will come up basically; when the relative humidity is higher than $95 \%$, it is very conducive to the growth of mold, and resulting in the rapid development of mildew. Moreover, the temperature also plays an important role on bamboo mildew, when the environmental temperature is below $15^{\circ} \mathrm{C}$ or above $35^{\circ} \mathrm{C}$, the mold grows slowly, and the optimum temperature for mold growth is between $20-30{ }^{\circ} \mathrm{C}$.

\section{Bamboo mold}

China has vast territory, the climate conditions are different. There are many kinds of bamboo species, and the mold populations are also different. In the 5 subdivisions of fungi, the types of fungi that damage bamboo timber are zygomycete subdivision and ascomycetes subdivision. Many kinds of fungi in the 4 subdivisions like basidiomycete's subdivision, adelomycete subdivision, etc. have damage on bamboo timber. The commonly seen molds that have the damage of mildew are Mucor, Penicillium, Trichoderma, Aspergillus and Zhipao mold.

Wu Kaiyun, etc. ${ }^{[3]}$ proposes that 15 kinds of molds may cause the mildew of Mao bamboo timber, among which 10 kinds are important moulding fungi, and they are Alternaria alternate, Aspergillus flavus, Penicilliumtrinum, P.chrysogenum, P . funiculosum, Chaetomium globosum, Fusarium pallidoroseum, F.camptoceras and Mucor hiemalis. Wu Guangjin, etc. ${ }^{[4]}$ study the molds on the Mao bamboo and Water bamboo from Yiyang and Changde of Hunan Province, and it is verified that there are 20 kinds, and 12 kinds appear commly: Penicillium chrysogenum, P.coryloPhilum, P.janthinellum, P.digitatum, P.expansum, Rhizopus nigricans, Aspergillus niger, Aspergillus flavus, Trichothecium roseum Fusarium 1Lactis, F.javanicum and F.moniliforme. Zhao Guihua, etc. ${ }^{[5]}$ separates and identifies 6 kinds of fungi from the mildew Mao bamboo and phyllestachys pubescens: Phoma necatrix, Alternaria viticola, A.restrictus, sphaeropsi siphotinae, Melanconum SP. and Macrophoma fabae. Wang Wenjiu, etc. ${ }^{[6-7]}$ study the mold and rot fungi of bamboo timber from Changning and Anning of Yunnan Peovince, and 52 kinds are identified: 3 kinds of zygomycete subdivisions, 3 kinds of ascomycetes subdivisions, 29 kinds of adelomycete subdivisions and 17 kinds of basidiomycetes subdivisions. Most of the molds belong to Hyphomycetes of adelomycete subdivision, among which the most typical ones are Penicillium Link of Hyphomycetaceae, Aspergillus (Mich), Trichoderma Pers, etc.

\section{Physiological characteristics of bamboo mold}

There are many kinds of fungi that would cause the mildew and rot of bamboo timber, only through understanding the categories and characteristics of bamboo timber mold that bamboo timber mildew can be prevented and effective ways can be taken. Wu Guangjin, etc. ${ }^{[4]}$ study the biological characteristics of part of the bamboo timber molds, it is believed that the spores of Fusarium will germinate when the relative humidity is $41 \%$, the spores of Penicillium corylophilum, Penicillium chrysogenum and Trichothecium roseum begin to terminate when the relative humidity is $63 \%$, the spore of Trichosporon will germinate when the relative humidity is $70 \%$, the germination rate of spore will increase with the increasing humidity, and the relative humidity of $93 \%$ is the most suitable humidity for the germination of each spore. The influence of temperature is: the optimum temperature of Fusarium is $20^{\circ} \mathrm{C} \sim 30^{\circ} \mathrm{C}$, the optimum temperature of Penicillium corylophilum, Penicillium chrysogenum and Trichothecium roseum is $15{ }^{\circ} \mathrm{C} \sim 30{ }^{\circ} \mathrm{C}$, and they cannot grow when the temperature is $35^{\circ} \mathrm{C}$. Ran Longxian, etc. ${ }^{[8]}$ study the biological characteristics of Aspergillus niger, Aspergillus flavus, Penicillium digitatum, Penicillium citrinum and Rhizopus nigricans which are all commonly seen molds, the influence of bamboo total soluble sugar and starch content on mold growth, and the relativity between the activity of the 5 kinds of molds' amylase and cellulase and the 
compression strength parallel to grain. The results show that the germination and growth of mold spore are the fastest when the temperature is $25{ }^{\circ} \mathrm{C} \sim 30{ }^{\circ} \mathrm{C}$ and the relative humidity is above $93 \%$, and none of the 5 kinds of mold spores will terminate or grow when the temperature is below $5{ }^{\circ} \mathrm{C}$ or the relative humidity is below $65 \%$; the optimum $\mathrm{PH}$ for growth is between 4 and 6 ; all the 5 kinds of molds can use starch or soluble sugar as carbon source, but only Aspergillus niger can grow on the medium that uses cellulose as carbon source; the total soluble sugar and starch content of bamboo are significant positive correlated with the growth of mold, all the 5 kinds of molds have amylase activity, and only Aspergillus niger has cellulase activity. The amylase activity of mold is negative correlated with the compressive strength parallel to grain of the rotten bamboo timber.

\section{Anti-mold methods of bamboo timber}

Chemical method. Chemical method is commonly seen in the anti-mold methods of bamboo timber, Song Ye, etc. ${ }^{[9]}$ propose that nanometer titanium dioxide not only has superior ability in absorbing $\mathrm{UV}$, but also can activate oxygen into reactive oxygen by using luminous energy, which can effectively destruct the structure of the molds absorbed on the surface, so it has enormous potential in improving the stability of material color and the property of anti-mold. Exploring at forming a thin film of nanometer titanium dioxide on the surface of bamboo timber at low temperature by using the sol - gel method, to significantly improve the stability of material color and the viability of anti-mold property. The study finds out that the thin film of nanometer titanium dioxide formed on the surface of bamboo timber is amorphous. After $120 \mathrm{~h}$ accelerated aging, the total color difference $\Delta \mathrm{E}$ of modified sample is about $1 / 16$ of the blank sample, which indicates that the stability of the bamboo timber color enhances significantly and the anti-mold property of modified bamboo also enhances to a certain extent.

Sun Fangli, etc. ${ }^{[10]}$ study the mildew performance of Phyllostachys pubescens after the treatment of cold water, hot water, benzene and alcohol, benzene and ethyl ether, $10 \mathrm{~g} / \mathrm{kg} \mathrm{HCl}$ and $10 \mathrm{~g} / \mathrm{kg} \mathrm{NaOH}$ solution. The results show that Trichoderma viride, Penicillium citrinum ans Aspergillus niger of bamboo chip have the best effect after being treated by $10 \mathrm{~g} / \mathrm{kg} \mathrm{HCl}$, the average infection values of samples are zero one month after being inoculated. The bamboo chip that is treated by $10 \mathrm{~g} / \mathrm{kg} \mathrm{NaOH}$ has relatively good anti-mold effect to Trichoderma and Penicillium citrinum. If the bamboo chip that is treated by $10 \mathrm{~g} / \mathrm{kg} \mathrm{NaOH}$ hasn't been washed by water, the average infection values are zero one month after being inoculated; the anti-mold effects will decrease in varying degrees after being washed by water, the average infection values of the samples of Trichoderma and Penicillium citrinum are 1.58 and 0.35 respectively. The bamboo chip that is treated by $10 \mathrm{~g} / \mathrm{kg} \mathrm{NaOH}$ has poor effect on the prevention of Aspergillus niger, and the sample average infection values of the treated bamboo to Aspergillus niger before and after being washed by water are 1.82 and 2.79 respectively. The bamboo chips that are treated by cold water, hot water, benzene and alcohol, benzene and ether all have poor anti-mold effect, the average infection values of samples all above 3.0 after 7 days, and it mainly due to different compositions and numbers in the bamboo timbers which are treated by different solutions.

Sun Fangli, etc. ${ }^{[1]}$ apply the newly cut Phyllostachys pubescensas of 3 to 4 years old as test materials, Penicillium, Trichoderma and Aspergillus niger are the test bacteria, comparing with the commonly used HBO3 and chlorothalonil, the anti-mold performance of the CCC and CZC of CMC is tested. The results show that the control effects of CCC and chlorothalonil on Trichoderma, Penicillium, Aspergillus niger is better than that of CZC, chitosan and boric acid; in comparison, the control effect of CCC on Penicillium is better than that of chlorothalonil, and the control effect of chlorothalonil on Trichoderma is better than that of CCC.

Nanometer method. Nanometer $\mathrm{TiO} 2$ is qualified with unique photocatalytic activity which can produce a large number of electron-hole pairs by absorbing ultraviolet radiation. These photo-electrons and holes have superior redox capacity which can kill many bacteria and viruses, and they also are qualified by wide range of antibacterial spectrum, good heat resistance, excellent antibacterial effect and so on, which may significantly improve the anti-bacterium and anti-mold properties of material. Sun Fengbo, etc. ${ }^{[12]}$ prepare $\mathrm{TiO} 2$ sol at low temperature by using the sol - gel 
method, and also accomplish the nano-TiO2 modification of bamboo timber by dip-coating technique. At the same time, they characterize the form and structure of $\mathrm{TiO}_{2}$ by using NMR, FESEM, XRD and EDAX, and focus on studying the influence of temperature on the morphology, the crystal form and the anti-bacterium and anti-mold properties of $\mathrm{TiO} 2 \mathrm{film}$. The results show that the TiO2 modified bamboo timber that are treated by the three kinds of temperature $\left(20,60,105^{\circ} \mathrm{C}\right)$ maintain the natural color, texture and structure of bamboo timber and the original non anti-bacteria property is changed into over $99 \%$ sterilizing rate to E. coli and the anti-mold property is increased by more than 10 times. This method is expected to be the new method of improving bamboo performance, and it also has reference meaning to the protection and improvement of other natural raw materials besides wood.

Microwave method. For the bamboo material that is treated by conventional methods, the egg and some mold spores with heat resistance cannot be completely killed, the survived mold will grow new mold points after a period of time, while for the bamboo material that is treated by microwave, all the worms and various kinds of molds are killed, and as the microwave penetrates into the material, all the eggs are killed, the bamboo will not have worms or mold even after several years. Cheng Wenzheng ${ }^{[13]}$ discusses the effect and mechanism of killing worms and molds inside bamboo material with micro energy through the comparison of a series of experimental test results, the experimental test results prove that microwave energy has good insecticidal effect and anti-mold effect, which solves the two quality problems of bamboo products fundamentally. Currently, the technology that adopts the microwave heating technology in drying, killing worms and preventing mold has been applied in industrial production.

Ray method. At present, ray radiation has been applied by many countries and regions in the processing of food, fruits and vegetables, which can achieve the purpose of sterilization and preservation, and this provides a reference for the application of ray radiation in the area of wood products preservation. Sun Fengbo, etc. ${ }^{[14]}$ carry on radiation processing on bamboo timber by the ray of ${ }^{60} \mathrm{Co}$ source, study the variation rule of the anti-mold performance of bamboo timber before and after the radiation, and also analyze the mechanism on how the anti-mold performance of bamboo timber is improved by ray. The results show that the anti-mold effect of bamboo timber is significantly increased with the increasing amount of radiation.

Biological method. Plant mildew preventive is safe to human and animals, because it can be degraded through photolysis, hydrolysis, and enzymolysis and so on. Bamboo vinegar stoste is the byproduct formed in the process of firing charcoal through pyrolysis, and bamboo vinegar liquid is a natural antibacterial agent and pesticide. Shen Zhehong, etc. ${ }^{[15]}$ study the antibacterial property of bamboo vinegar stoste and bamboo vinegar preparations on wood mold, in order to expand the use of bamboo vinegar liquid and develop a bamboo protective agent with high efficiency and low toxicity. The results show that the antibacterial rate of bamboo vinegar stoste to the mold of three kinds of wood is $99 \%$, but it does not have anti-mold property. According to this, three bamboo vinegar preparations of PW1, PW2, PW3 are researched and developed, their antibacterial property is significantly enhanced; the anti-mold performance of the bamboo timber that is treated by bamboo vinegar preparations is significantly improved.

\section{Prospect on the anti-mold research of bamboo timber}

As there is a large number of mildew and rot fungi of bamboo timber and their distribution varies with the environmental changes, the difficulty and workload of the study on them are considerable. At present, although there are many studies on the mildew fungi of bamboo timber and the understanding is also relatively clear, but stain fungi and rot fungi are rarely known by people, the boundaries of mold, stain fungi and rot fungi and the boundaries of brown rot, white rot, soft rot are still not very clear. It leads to more studies on the anti-mold research of bamboo timber and less studies on the anti-corrosion and anti-discoloration that are equally important to anti-mold. Furthermore, the bacteria applied in the anti-mold and anti-corrosion experiments may not be typical and appropriate, which may affect the reliability of the results. 
The species of the mildew and rot fungi of bamboo timber also differ because of some natural conditions like geography, climate and so on, even the major species of hazardous fungi also have a great difference in the study results of various areas. A large amount of painstaking work is needed to be done, in order to clarify the species and distribution situation of the major mildew fungi in different regions and climatic zones.

The anti-mold of bamboo timber is a complex project, it involves bamboo science, botany, microbiology, chemistry and so on, in order to research and develop new treatment method and drug of anti-mold, the relation of bamboo mold, bamboo timber and external environment, the parasitic conditions of different fungi inside the bamboo timber and the inhibition mechanism of mildew preventive to mold growth need to be emphasized. Furthermore, the research on the mechanism of the mildew, decay, discoloration, infestation, etc. of bamboo timber need to be strengthened, and the research on the mechanics, durability, pest control, anti-mold, anti-corrosion and some other performances before and after the anti-mold and anti-corrosion and the corresponding testing and evaluation methods also need to be strengthed.

The future research direction of the anti-mold and anti-corrosion of bamboo timber will focus on the development of new, green, highly effectively and inexpensive mold preservatives, which mainly includes anti-mold and anti-corrosion effect, durability, cost, environmental protection, anti-loss, the influence on the physical and mechanical properties of plates and so on.

\section{Acknowledgment}

The research of this dissertation is supported by Youth Scientific Research Foundation of Central South University of Forestry and Technology of China (No.2010007A).

\section{References:}

[1] Liu Yuan, Lie Changming, Chen Jianhua, Bamboo Timber Mildew and Anti-mold Treatment [J], Hunan Forestry Science and Technology, 1993, 20(2):61 62.

[2] Weng Yuexia,Wu Kaiyun, Studies On Biology Of Bamboo Timber Moulding [J]. Forest Research, 1991, 4(1):17 20.

[3] Wu Kaiyun, Weng Yuexia, Bamboo Mildew-rotting and Its Relation with Environmental Condition [J], Forest Research,2000,13(1): 63 70.

[4] Wu Guangjing, Lin Xuejian, Ran Longxian, Identifiaton of Moulds Infecting Bamboo Wood and Formulation of Fungicidal Compounds [J], Economic Forest Reseaches, 1994,12(2):50 55.

[5] Jin Zhongwei, Zhao Guihua, He Wenlong, Studies On The Fungi On The Bamboo Wood [J], Journal Of Nanjing Forestry Unversity,1994,18(3):87 90.

[6] Fu Hui, Chen Yuhui, Wang Wenjiu, etc., Studies on Mouldy Fungi and Their Characteristics of Five Kinds of Timber Bamboo in Yunnan [J], Journal of Bamboo Research, 1999,18(1):16 22.

[7] Wang Wenjiu, Hui Chaomao, Chen Yuhui, Mildew and Rot of Bamboo Wood and Mold Fungi [J], Journal of Bamboo Research,2000. 19 (2) :1-6.

[8] Ran Longxian, Wu Guangjing, Lin Xuejian, Physiological Characteristics of Moulds Infecting Bamboo Wood and Mould Control[J], Journal of Central South Forestry Universith,1997,17(2):14 19.

[9] Song Ye, Wu Yiqiang, Yu Yan, Effect of Titanium Dioxide on Color Stability and Mildewproof Properties of Bamboo [J]. Journal Of Bamboo Research, 2009, 28(1):30 34.

[10]Sun Fangli, Mao Shengfeng, Wen Guifeng, etc., Anti-mold Effects of Bamboo Timber Treated with Different Solutions [J], Journal of Zhejiang Forestry College, 2006, 23(2): 135 139. 
[11]Sun Fangli, Duan Xinfang, Wen Guifeng, etc., Anti-mold Effects of CMC Wood Preservatives on Bamboo Wood [J], Scientia Silvae Sinicae, 2006, 42(3):40 43.

[12]Sun Fengbo, Yu Yan, Jiang Zehui, etc., Nano TiO_2 Modification of Bamboo and Its Antibacterial and Mildew Resistance Performance [J], Spectroscopy and Spectral Analysis, 2010, 30(4):1056 1060.

[13]Cheng Wenzheng, Ye Yuhuang, On the Effects of Microware Power Use in Killing Bamboo Worm and Preventing Mould [J], Journal of Fuzhou University,1999,27(5):28 30.

[14]Sun Fengbo, Jiang Zehui, Fei Benhua, etc., Effect of $\gamma$-Ray Application on Bamboo Mold Resistance [J], China Wood Industry,2011,25(3):23 25.

[15]Shen Zhehong, Fang Qun, Ye Liangming, etc., Bamboo Vinegar Antifungal Performance and Mold Inhibition [J], China Wood Industry, 2009, 23(6):37 40. 